\title{
Ribavirin in Acute Hepatitis E Infection in Patients with Gynecological Cancer: A Case Series
}

\author{
Dominik Bettinger ${ }^{* 1,2}$, Stefan Schlabe ${ }^{\# 3,4}$, Sven Pischke ${ }^{5}$, Michael R. Mallmann7 , \\ Mignon-Denise Keyver-Paik ${ }^{4,6}$, Walther Kuhn ${ }^{4,6}$, Christian P. Strassburg ${ }^{3,4}$, \\ Robert Thimme ${ }^{\neq 1}$ and Ulrich Spengler ${ }^{\neq 3,4}$
}

\begin{abstract}
${ }^{1}$ Department of Medicine II, Medical Center University of Freiburg, Faculty of Medicine, University of Freiburg, Freiburg, Germany; ${ }^{2}$ Berta-Ottenstein-Programme, Faculty of Medicine, University of Freiburg, Freiburg, Germany; ${ }^{3}$ Department of Internal Medicine I, University of Bonn, Bonn, Germany; ${ }^{4}$ Center for Integrated Oncology Cologne/Bonn, Bonn, Germany; ${ }^{5}$ Department of Medicine, University Medical Center Hamburg-Eppendorf, Hamburg, Germany; ${ }^{6}$ Department of Obstetrics and Gynecology, University of Bonn, Bonn, Germany; ${ }^{7}$ Department of Obstetrics and Gynecology, University of Cologne, Cologne, Germany
\end{abstract}

\section{Abstract}

Hepatitis E virus infection is usually a self-limited disease. However, during the last years there has been growing evidence for prolonged and chronic infection occurring in patients with immunosuppression. Also patients with malignant and rheumatic diseases have been identified to be at risk for chronic hepatitis E. However, their course and prognosis are not well characterized and there have been no reports of hepatitis $E$ virus infection in patients with gynecological cancer. Here, we report three Caucasian females with breast and ovarian cancers presenting with elevation of aminotransferase levels during anticancer treatment. Although only few or no clinical hints suggested hepatitis $E$ virus infection, the diagnosis of hepatitis $E$ virus infection was confirmed by seroconversion, which might occur with some delay, and/or by polymerase chain reaction. While two patients had a selflimited course, the third patient with a high-risk oncological constellation required ribavirin in order to resume chemotherapy. These cases highlight the need for hepatitis $\mathrm{E}$ virus testing in patients with gynecological cancer and elevated aminotransferase levels. Further, these cases show that in selected high-risk patients, ribavirin treatment may be necessary based on the decision of a multidisciplinary team.

Citation of this article: Bettinger $D$, Schlabe S, Pischke S, Mallmann MR, Keyver-Paik MD, Kuhn W, et al. Ribavirin in Acute Hepatitis E Infection in Patients with Gynecological Cancer: A Case Series. J Clin Transl Hepatol 2018;6(2):237-240. doi: 10.14218/JCTH.2017.00063.

Keywords: Hepatitis E; Gynecological cancer; Liver function test; Chemotherapy; Immunosuppression.

Abbreviations: ALT, alanine aminotransferase; ASH, alcoholic steatohepatitis; AST, aspartate aminotransferase; CT, computed tomography; GGT, $\gamma$-glutamyltransferase; HEV, hepatitis E virus; HIV, human immunodeficiency virus; INR, international normalized ratio; NASH, nonalcoholic steatohepatitis; PCR, polymerase chain reaction; SOS, sinusoidal obstruction syndrome.

Received: 4 October 2017; Revised: 23 November 2017; Accepted: 14 December 2017

${ }^{\#}$ These authors contributed equally to this work.

${ }^{\ddagger}$ These authors shared equal senior authorship.

*Correspondence to: Dominik Bettinger, Department of Medicine II, Medical Center University of Freiburg, Hugstetter Str. 55, Freiburg 79106, Germany. Tel: +49-761-270-32440, Fax: +49-761-270-32440, E-mail: dominik.bettinger@ uniklinik-freiburg.de

\section{Introduction}

For decades, hepatitis E virus (HEV) infection has been considered to be a self-limiting acute tropical disease. ${ }^{1,2}$ Recently, this interpretation has been changed as HEV infection is not restricted to the tropics but is also endemic in the Western world ${ }^{1}$ with different distribution of the four HEV genotypes also showing different transmission routes. HEV genotypes 1 and 2 are characteristic infections in tropical countries, that are transmitted by contaminated drinking water. In contrast, HEV genotypes 3 and 4 are endemic in industrialized nations, being transmitted via porcine meat or blood products. ${ }^{1,2}$ Importantly, chronic HEV infection can occur in immunosuppressed individuals (i.e. solid organ recipients, human immunodeficiency virus (HIV)-infected patients, patients with lymphoma and those after stem cell transplantation). However, the prevalence and importance of HEV infection in patients with solid cancer is unclear. Indeed, elevated liver function tests in patients with solid cancer during chemotherapy comprise a broad spectrum of differential diagnoses, but acute HEV infection is not regularly considered. Here, we describe three patients with breast and ovarian cancer who acquired acute HEV infection and presented with elevated aminotransferase levels during chemotherapy.

\section{Case report}

\section{Case 1}

This 34 year-old Caucasian female received adjuvant chemotherapy to treat bilateral BRCA-1-positive breast cancer. Elevated aminotransferase levels were detected during chemotherapy and lead to referral to the Hepatology Department of the University Hospital of Bonn for multidisciplinary management of her hepatitis (Fig. 1A). The patient had bilateral invasive G3 breast cancer (right side: invasive ductal carcinoma, pT1cpN0(0/1,sn-)MOROLOV0G3, triple-negative; left side: metaplastic carcinoma, pT1cpN2(9/14,ece+,sn+) MOROLOVOG3, triple-negative). Metastases were excluded and bilateral tumors surgically removed and axillary lymph nodes were dissected without complications. 
A

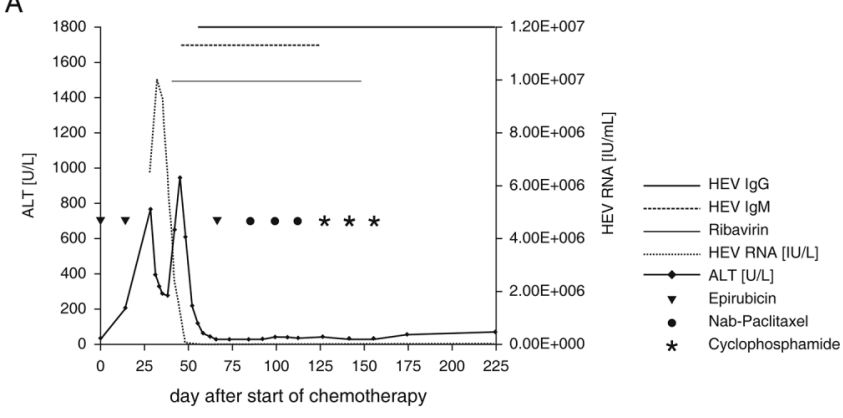

B

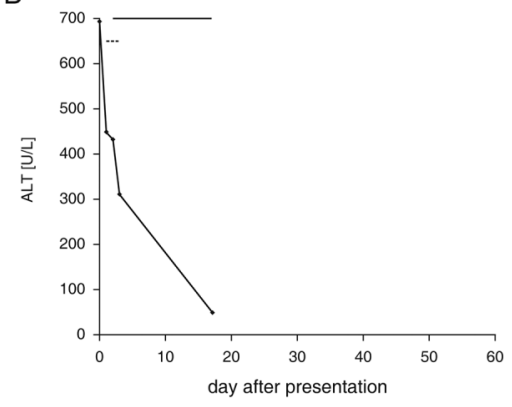

C

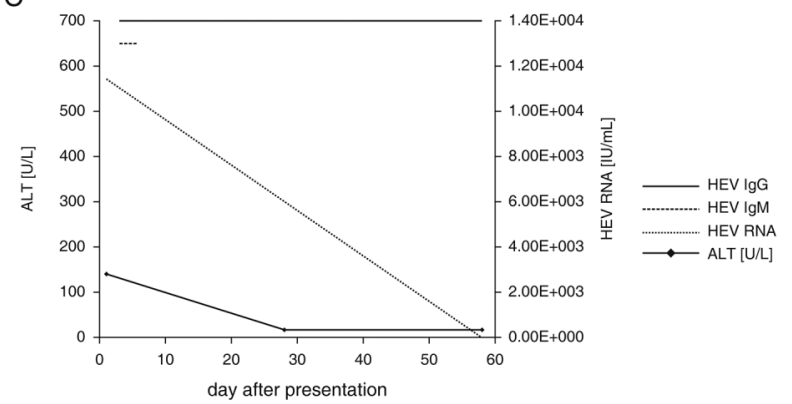

Fig. 1. Course of aminotransferases in patients with gynecological cancer and HEV infection. (A) Case 1 developed hepatitis during chemotherapy for treatment of high-risk breast cancer. The HEV viral load showed a remarkable increase during chemotherapy and was cleared rapidly with ribavirin treatment. Seroconversion took place with delay. (B) Case 2 developed hepatitis 2 years after chemotherapy with ongoing bevacicumab therapy for treatment of ovarian cancer. After spontaneous clearance of the HEV infection, ALT levels decreased continuously. (C) Case 3 developed hepatitis 1 year after radiotherapy and ongoing tamoxifen treatment for treatment of breast cancer. Spontaneous clearance of HEV with low viral load and subsequent normalization of ALT occurred.

This high-risk patient was included in the GAIN-2 study and allocated to receive adjuvant chemotherapy with epirubicin, nanoparticle albumin-bound-paclitaxel and cyclophosphamide. A first cycle of epirubicin $\left(150 \mathrm{mg} / \mathrm{m}^{2}\right)$ was started at 5 weeks after surgery. Aminotransferase levels were still normal (alanine aminotransferase (ALT) $27 \mathrm{U} / \mathrm{L}$ ). Two weeks later, when a second dose of epirubicin was applied, the patient complained of severe nausea, and elevated ALT levels $(201 \mathrm{U} / \mathrm{L})$ were first attributed to drug toxicity. Aminotransferase levels continued to rise (ALT $763 \mathrm{U} / \mathrm{L}$ ) and the third epirubicin dose was deferred. Ultrasonography of the liver ruled out liver metastasis, cholestasis and portal thrombosis. Viral testing revealed high HEV replication $(6,485,714 \mathrm{U} / \mathrm{mL})$. Polymerase chain reactions (PCRs) performed 4 days and 7 days later yielded high viral load beyond the limit of quantification $(>10,000,000 \mathrm{IU} / \mathrm{mL})$, both in blood and stool. In order to shorten the duration of high-level HEV replication and to avoid prolonged interruption of adjuvant chemotherapy in this high-risk patient, antiviral therapy with $400 \mathrm{mg}$ ribavirin bd was initiated (Fig. 1A).

Ribavirin was well tolerated without significant decreases in hemoglobin. Serology revealed appearance of HEV-IgM and of IgG 17 days and 28 days after HEV diagnosis, respectively. HEV replication decreased continuously and the patient tested negative 6 weeks after diagnosis. After ALT levels had normalized (26 U/I) and HEV PCR became negative, chemotherapy was resumed. We continued ribavirin during the next three cycles of nanoparticle albuminbound paclitaxel and finally stopped antiviral therapy after the second cyclophosphamide cycle. Throughout the further course of chemotherapy, liver enzymes remained consistently normal and HEV replication remained undetectable.

\section{Case 2}

A 29 year-old Caucasian female developed persisting abdominal pain and presented at the Gastroenterology Department at the University Hospital Freiburg 3 days later. Two years before, she had undergone hysterectomy, omentectomy, colectomy and resection of the rectum with dissection of pelvine and paraaortic lymph nodes for treatment of ovarian cancer. After surgery, she had received chemotherapy with 8 cycles of carboplatin and paclitaxel. On admission to our department, the patient was treated with bevacizumab according to the GOG-218 study. ${ }^{3}$

Clinical examination of the patient confirmed pain over the left upper abdominal quadrant. Changes in stool habits or gastrointestinal symptoms were not reported. Laboratory analyses revealed elevated aminotransferases (ALT $692 \mathrm{U} / \mathrm{L}$, aspartate aminotransferase (AST) $138 \mathrm{U} / \mathrm{L}$ ) as well as $\gamma$-glutamyltransferase (GGT) levels (550 U/L) (Fig. 1B). Ultrasound imaging excluded intra- or extrahepatic cholestasis, choledocholithiasis, cholecystitis, portal vein thrombosis and Budd-Chiari-syndrome. Given her history of abdominal surgery and aggravating clinical symptoms, an abdominal computed tomography (CT) scan and upper gastrointestinal endoscopy was performed, which did not reveal any significant new findings.

Since laboratory analyses suggested hepatitis, serological testing for hepatitis A, B, C and E viruses as well as EpsteinBarr virus and cytomegalovirus infections was performed yielding elevated anti-HEV IgM and IgG levels as markers of acute HEV infection. In blood and stool, the HEV PCR was negative, indicating that the patient had already spontaneously cleared the HEV infection. Abdominal pain was controlled with analgesics and the patient was discharged in a good condition.

\section{Case 3}

A 67 year-old Caucasian female presented to the Gynecological Department at the University Hospital Bonn for elective laparoscopy of an ovarian cyst. One year before she had been diagnosed with left-sided breast cancer ( $\mathrm{pT} 2 \mathrm{pNO}(0 / 3 \mathrm{sn}-$ ) LOVOROMOG2, ER9/12, PR 7/12, Ki67 10\%, HER-2neu negative) that was treated with left upper quadrant resection. Sentinel lymph nodes were negative and there was no evidence of metastatic disease in imaging studies. Thus, chemotherapy was not recommended in this low-risk 
situation. Instead, the patient received systemic tamoxifen (20 mg once daily) and radiotherapy (60 Gy).

CT staging identified a left ovarian cyst $(5.7 \mathrm{~cm} \times 6.7 \mathrm{~cm}$ in diameter) but surgical exploration was deferred for 1 year. Prior to anesthesia and laparoscopic adenectomy, which revealed serous ovarian cystadenoma elevated aminotransferase levels (ALT 140 U/L, AST 107 U/L, GGT 224 U/L) were noted (Fig. 1C). Virological testing identified acute HEV infection (genotype 3, viral load $11249 \mathrm{IU} / \mathrm{L}$ in blood, $21714 \mathrm{IU} / \mathrm{L}$ in stool) in the presence of positive HEV IgM and IgG serology. Based on the patient's clinical stability and only mildly elevated aminotransferase levels, antiviral treatment was not started. Aminotransferase levels normalized (ALT $18 \mathrm{U} / \mathrm{L}$, AST $19 \mathrm{U} / \mathrm{L}$, GGT $51 \mathrm{U} / \mathrm{L}$, bilirubin $0.56 \mathrm{mg} / \mathrm{dL}$ ) within 4 weeks and both HEV replication and HEV IgM became negative. Throughout her hepatitis, the patient did not display any symptoms or clinical signs of acute hepatitis.

\section{Discussion}

HEV infection in immunosuppressed individuals is a topic of emerging relevance, since hepatitis $E$ has been described in solid organ and stem cell transplant recipients and HIVinfected patients. ${ }^{4-6}$ Furthermore, within the last months, hematological and rheumatological patients were recognized to be at risk for HEV infection. ${ }^{7,8}$ Here, we demonstrate that HEV infection must be considered as a differential diagnosis in patients with gynecological cancer presenting with elevated aminotransferases during antitumor treatment.

Similar to transplant recipients under immunosuppression, in whom serology may fail to detect $\mathrm{HEV}^{9}$ serology was not reliable in our cases. In patient 1 , seroconversion to antiHEV IgM and IgG could only be observed with a delay of more than 2 weeks after first detection by PCR, indicating not to rely on serological diagnosis but to confirm diagnosis by PCR. Being aware of the highly various immune status of patients receiving chemotherapy and the possibility that serology fails to accurately detect HEV infection, we propose to perform HEV serology as well as HEV PCR in these special patients when HEV infection is suspected.

Patient 2 was diagnosed after the viremic period. Patient 3 who presented with only slightly elevated aminotransferase levels in the presence of antibodies was considered to have control of her infection. Thus, antiviral treatment was not initiated and spontaneous HEV resolution was confirmed during follow-up. In contrast, patient 1 was treated with ribavirin to facilitate early continuation of chemotherapy due to an oncological high-risk constellation. High-level HEV replication both in blood and stool 2 weeks after her second cycle of chemotherapy led to suspicion of a high risk for chronic infection due to immunosuppression. Importantly, ribavirin treatment lead to a rapid clearance of HEV. Nevertheless, treatment with ribavirin is still discussed controversially, in particular with respect to its optimum dose and treatment duration.

To date, no guidelines exist concerning the optimal dose of ribavirin in these special patients. Pischke et al. ${ }^{10}$ described an HEV patient in whom the dose of ribavirin was reduced to $200 \mathrm{mg}$ daily. However, in this patient viral rebound occurred and viral resistance was suspected. In the patients described by Pischke et al.,10 a dose of $600 \mathrm{mg}$ to $1000 \mathrm{mg}$ daily was applied according to the patient's comorbidities and the hemoglobin levels. ${ }^{10}$ In our patient, the dose of $800 \mathrm{mg}$ was used, as we wanted to clear the infection as early as possible in order to continue chemotherapy and to avoid development of resistance. But, we were also aware of the risk of anemia, which may have been aggravated by the applied chemotherapy. This was the main reason why we did not start with a higher daily dose of $1000 \mathrm{mg}$.

It remains unclear how long ribavirin should be given and when chemotherapy can be safely restarted. In our patient with a high need for antitumor treatment, chemotherapy was resumed after aminotransferase levels had returned to baseline. We planned to continue ribavirin treatment until the end of chemotherapy, but the patient decided to stop ribavirin when the HEV PCR had become negative and no relapse occurred during continuation of chemotherapy. This may be the rationale for discontinuation of ribavirin treatment when HEV PCR becomes negative in stool and blood. Although to date, prospective, double-blind, placebo-controlled studies focusing on the efficacy, safety and duration of ribavirin in HEV infection do not exist, our report and a large retrospective analysis in transplant recipients as well as other single center experiences seem to indicate that ribavirin is safe and efficient in this setting. ${ }^{10,11}$

Diagnosis of HEV infection is difficult, as symptoms can vary considerably. This was confirmed in our patients, who were either asymptomatic or presented with nonspecific complaints (i.e. abdominal pain, nausea). The differential diagnosis of elevated aminotransferase levels in patients with solid cancers and chemotherapy comprises a broad spectrum of possible underlying causes (Fig. 2). Importantly, it is often difficult to clearly assess the main reason for elevated liver enzymes in these patients. In patient 1 , we observed two peaks of aminotransferases. The first peak was primarily associated with drug toxicity. After chemotherapy was stopped, the liver enzymes did not decrease sufficiently, and after another increase further investigations were started and HEV infection was revealed. Probably, liver toxicity led to a first liver damage, which made the patient susceptible for HEV infection. However, as we did not perform liver biopsy in this patient, we cannot prove this hypothesis.

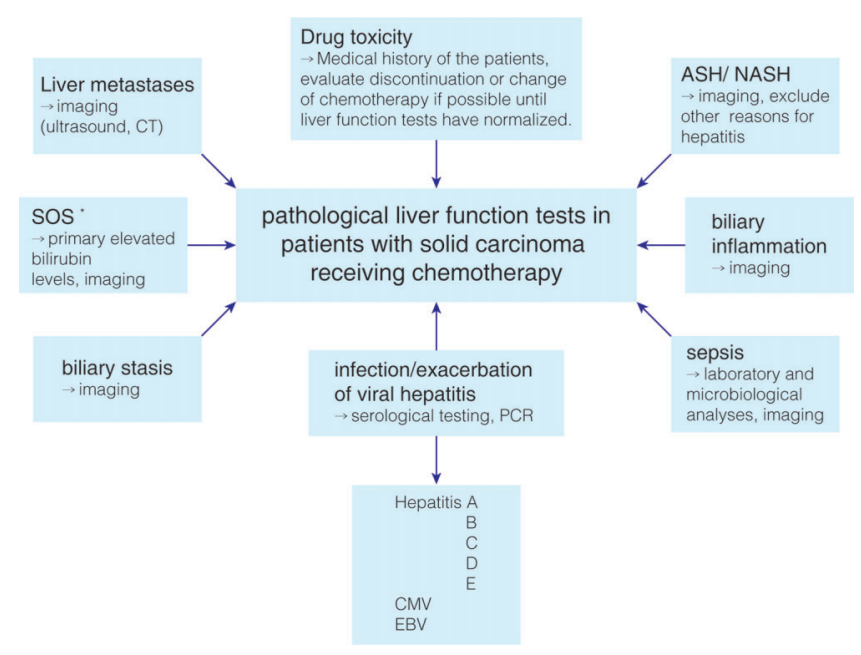

Fig. 2. Pathological liver function tests and hepatic adverse events in patients with solid carcinoma receiving chemotherapy. *SOS, sinusoidal obstruction syndrome, some chemotherapeutic agents, for example oxaliplatin, can cause sinusoidal necrosis leading to SOS. Abbreviations: ASH, alcoholic steatohepatitis; $\mathrm{NASH}$, nonalcoholic steatohepatitis. 
In addition to exacerbation of hepatitis $\mathrm{C}$ virus and reactivation of latent hepatitis $B$ virus infection, ${ }^{12,13}$ patients receiving chemotherapy are prone to acquire further bacterial and viral infections since their adoptive immunity is severely compromised. Thus, analogous to other immunosuppressed patients, HEV infection can be acquired by contaminated food during chemotherapy of solid malignant tumors. Moreover, tumor patients often need blood products, which constitutes another possible route of HEV transmission that can lead to acute $\mathrm{HEV}^{1}$ and progression to chronic HEV infection in the setting of immunosuppression. ${ }^{14,15}$ In this context, it should be critically discussed if blood donors and blood products should also be checked routinely for the presence of HEV contamination.

Of note, however, our patients did not receive blood products. After having excluded blood products as the transmission route and previous trips to HEV endemic countries, we retrospectively tried to assess the patients' eating history. Our retrospective assessment was not able to identify special eating habits. However, we have to assume food-born transmission as the most probable reason for HEV infection in the Western world. Therefore, our cases highlight that it is necessary to inform patients receiving chemotherapy about the risk of HEV transmission in undercooked meat, so that these patients develop an awareness of this probable risk.

\section{Conclusions}

In conclusion, our results clearly indicate that patients with the onset of hepatitis during chemotherapy must be tested for HEV infection. The diagnosis should be based on HEV PCR because antibody formation is delayed, and depending on the time point during the course of acute infection HEV test results might be falsely negative. Finally, the need for ribavirin in confirmed acute HEV infection must be evaluated considering the risk of tumor progression/recurrence and the level of immunosuppression induced by chemotherapy and the malignant disease itself.

\section{Acknowledgments}

Dominik Bettinger is supported by the Berta-OttensteinProgramme, Faculty of Medicine, University of Freiburg.

\section{Conflict of interest}

The authors have no conflict of interests related to this publication.

\section{Author contributions}

Treatment of patient (hepatology), acquisition of data, analysis, drafting of the manuscript (DB), treatment of patient (hepatology), acquisition of data, drafting of the manuscript (SS), drafting of the manuscript, critical revision of the manuscript for important intellectual content (SP), treatment of the patient (gynecology), critical revision of the manuscript for important intellectual content (MRM), treatment of the patient (gynecology), critical revision of the manuscript for important intellectual content (MDKP), critical revision of the manuscript for important intellectual content (WK), drafting of the manuscript, critical revision of the manuscript for important intellectual content (CPS), drafting of the manuscript, treatment of patient (hepatology), critical revision of the manuscript for important intellectual content (RT), drafting of the manuscript, treatment of patient (hepatology), critical revision of the manuscript for important intellectual content (US).

\section{References}

[1] Pischke S, Behrendt P, Bock CT, Jilg W, Manns MP, Wedemeyer H. Hepatitis E in Germany-an under-reported infectious disease. Dtsch Arztebl Int 2014; 111:577-583. doi: 10.3238/arztebl.2014.0577.

[2] Hoofnagle JH, Nelson KE, Purcell RH. Hepatitis E. N Engl J Med 2012;367: 1237-1244. doi: 10.1056/NEJMra1204512.

[3] Burger RA, Brady MF, Bookman MA, Fleming GF, Monk BJ, Huang $\mathrm{H}$, et al. Incorporation of bevacizumab in the primary treatment of ovarian cancer. N Engl J Med 2011;365:2473-2483. doi: 10.1056/NEJMoa1104390.

[4] Kamar N, Selves J, Mansuy JM, Ouezzani L, Péron JM, Guitard J, et al. Hepatitis $\mathrm{E}$ virus and chronic hepatitis in organ-transplant recipients. $\mathrm{N}$ Engl J Med 2008;358:811-817. doi: 10.1056/NEJMoa0706992.

[5] Versluis J, Pas SD, Agteresch HJ, de Man RA, Maaskant J, Schipper ME, et al. Hepatitis $E$ virus: an underestimated opportunistic pathogen in recipients of allogeneic hematopoietic stem cell transplantation. Blood 2013;122:10791086. doi: 10.1182/blood-2013-03-492363.

[6] Dalton HR, Bendall RP, Keane FE, Tedder RS, Ijaz S. Persistent carriage of hepatitis E virus in patients with HIV infection. N Engl J Med 2009;361:10251027. doi: 10.1056/NEJMc0903778.

[7] Tavitian S, Péron JM, Huynh A, Mansuy JM, Ysebaert L, Huguet F, et al. Hepatitis $E$ virus excretion can be prolonged in patients with hematological malignancies. J Clin Virol 2010;49:141-144. doi: 10.1016/j.jcv.2010.06.016.

[8] Bauer H, Luxembourger C, Gottenberg JE, Fournier S, Abravanel F, Cantagrel $A$, et al. Outcome of hepatitis $E$ virus infection in patients with inflammatory arthritides treated with immunosuppressants: a French retrospective multicenter study. Medicine (Baltimore) 2015;94:e675. doi: 10.1097/MD. 0000000000000675.

[9] Pischke S, Suneetha PV, Baechlein C, Barg-Hock H, Heim A, Kamar N, et al. Hepatitis $\mathrm{E}$ virus infection as a cause of graft hepatitis in liver transplant recipients. Liver Transpl 2010;16:74-82. doi: 10.1002/It.21958.

[10] Pischke S, Hardtke S, Bode U, Birkner S, Chatzikyrkou C, Kauffmann W, et al. Ribavirin treatment of acute and chronic hepatitis $\mathrm{E}$ : a single-centre experience. Liver Int 2013;33:722-726. doi: 10.1111/liv.12114.

[11] Kamar N, Izopet J, Tripon S, Bismuth M, Hillaire S, Dumortier J, et al. Ribavirin for chronic hepatitis $\mathrm{E}$ virus infection in transplant recipients. $\mathrm{N}$ Engl J Med 2014;370:1111-1120. doi: 10.1056/NEJMoa1215246.

[12] Lau GK. Hepatitis B reactivation after chemotherapy: two decades of clinical research. Hepatol Int 2008;2:152-162. doi: 10.1007/s12072-008-9056-3.

[13] Mahale P, Kontoyiannis DP, Chemaly RF, Jiang Y, Hwang JP, Davila M, et al. Acute exacerbation and reactivation of chronic hepatitis $C$ virus infection in cancer patients. J Hepatol 2012;57:1177-1185. doi: 10.1016/j.jhep.2012. 07.031.

[14] Kamar N, Garrouste C, Haagsma EB, Garrigue V, Pischke S, Chauvet C, et al. Factors associated with chronic hepatitis in patients with hepatitis $\mathrm{E}$ virus infection who have received solid organ transplants. Gastroenterology 2011;140:1481-1489. doi: 10.1053/j.gastro.2011.02.050.

[15] Wang Y, Zhou X, Debing Y, Chen K, Van Der Laan LJ, Neyts J, et al. Calcineurin inhibitors stimulate and mycophenolic acid inhibits replication of hepatitis $\mathrm{E}$ virus. Gastroenterology 2014;146:1775-1783. doi: 10.1053/j.gastro.2014. 02.036 . 